\title{
Detailed Atomic Structure of Defects in 2D Materials: From Graphene to Transition Metal Dichalcogenides.
}

\author{
Jamie H. Warner ${ }^{1}$ \\ ${ }^{1 .}$ Department of Materials, University of Oxford, OX13PH, Oxford, UK
}

Understanding the exact details of the position of atoms within defective regions in materials helps model and predict their properties accurately. Two dimensional materials that are only one atom thick, such as graphene and boron nitride, make identifying the positions of single atoms substantially easier than from 3D material structures. Aberration-corrected transmission electron microscopy at low accelerating voltages of $80 \mathrm{kV}$ and below, provides high atomic contrast in both phase contrast TEM imaging as well as in annular dark field scanning transmission electron microscopy. In recent work we have shown how monochromation of the electron source reduces the energy spread and lowers the detrimental effects of chromatic aberration on spatial resolution. The combination of monochromation and image correction in phase contrast HRTEM mode at an accelerating voltage of $80 \mathrm{kV}$ yields images of graphene with contrast spots associated with the position of individual atoms, as shown in figure 1, which when fitted with Gaussian profiles can give FHWM's of 80pm, indicating that sub-Angstrom resolution is approached. This led to the ability to fully resolve the position of individual carbon atoms in graphene around defects and measure the changes in $\mathrm{C}-\mathrm{C}$ bond lengths. Correlating the atomic structural models deduced from HRTEM with density functional theory provided insights into how changes in charge density around defect clusters causes bond elongation and compression [1,2]. In new work this has been extended to high temperature measurements by using an in-situ heating holder with suspended graphene samples. Totally different defect structures are produced at these high temperatures $\left(>600{ }^{\circ} \mathrm{C}\right)$ and are likely caused by changes to the flexibility of graphene and limits to out-of-plane distortions that help accommodate strain at low temperatures.

In more recent work we have extended our ultra-high spatial resolution phase contrast HRTEM imaging to studying defects in the transition metal dichalcogenide 2D crystal, $\mathrm{MoS}_{2}$, shown in figure 2 . Monolayered $\mathrm{MoS}_{2}$ is three atoms thick and large defect structures are far more complex than in graphene and more challenging to accurately resolve. Sulfur atoms originally in pristine $2 \mathrm{H}$ phase of $\mathrm{MoS}_{2}$ can flip positions, adopting 1T like locations, within defect structures to help stabilize Mo atoms. Detecting the contrast of $\mathrm{S}$ atoms in 1T like positions requires spatial resolution approaching Angstrom level. The ability to accurately resolve these features requires spatial resolutions close to the Angstrom level and demonstrates the benefit of the higher spatial resolution achieved by monochromation of the electron source. Time dependent images of the migration of single vacancies into line defects is studied and the flipping of $\mathrm{S}$ atoms to new $1 \mathrm{~T}$ positions detected.

\section{References:}

[1] J. H. Warner, E. R. Margine, M. Mukai, A. W. Robertson, F. Giustino, A. I. Kirkland, Science, (2012) 337, pp. 209-212.

[2] J. H. Warner, G-D. Lee, K. He, A. Robertson, E. Yoon, A. I. Kirkland, ACS Nano (2013), 7, pp 9860-9866.

[3] The author thanks the funding from the Royal Society. 


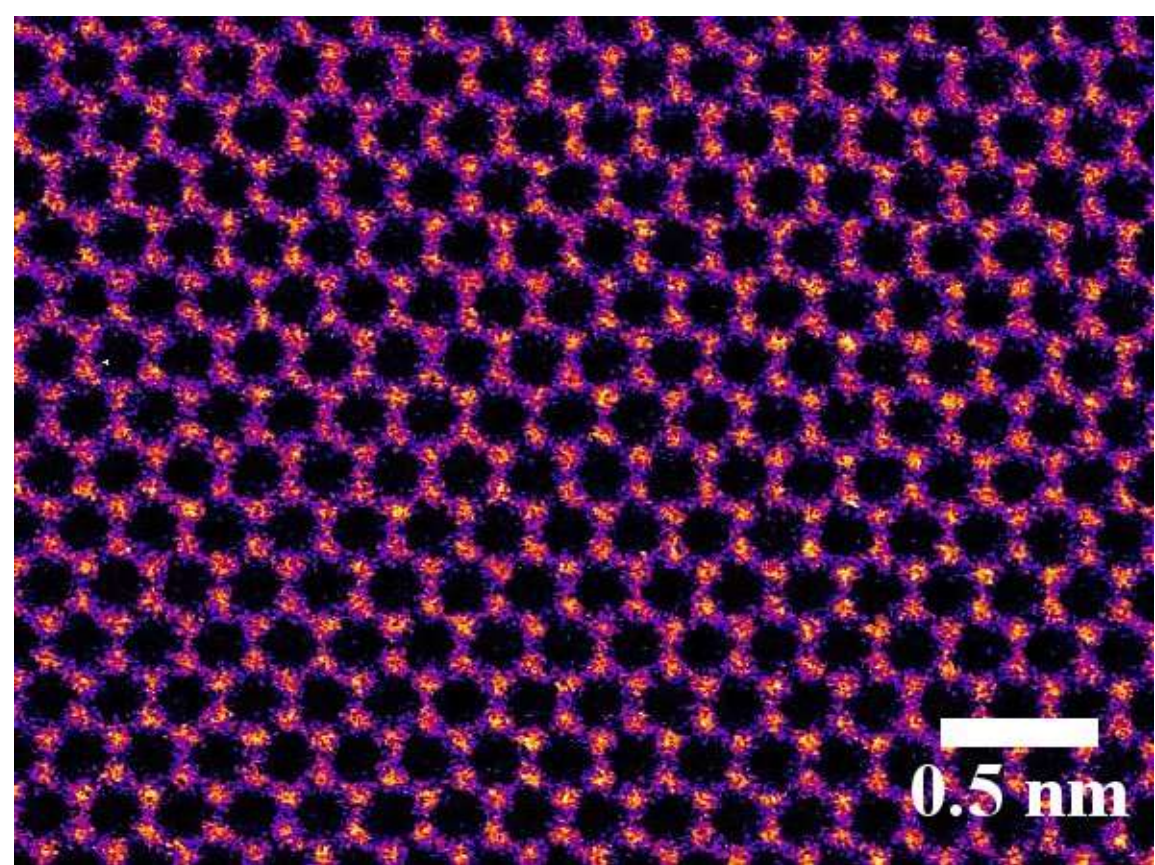

Figure 1. Aberration corrected transmission electron microscopy image of monolayer graphene at an accelerating voltage of $80 \mathrm{kV}$ and with monochromation of the electron source.

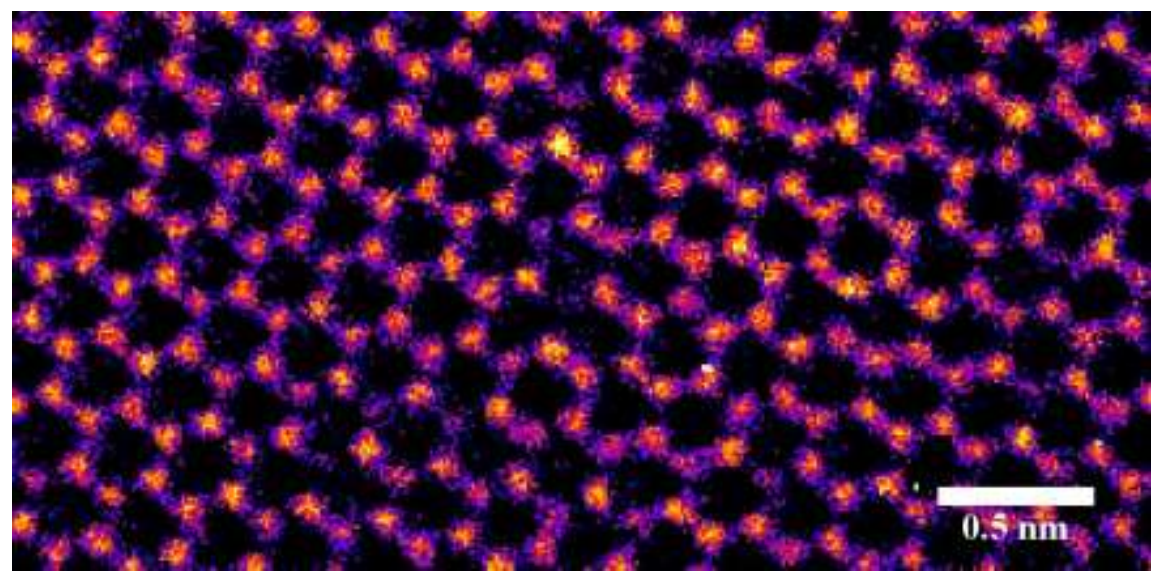

Figure 2. Aberration-corrected transmission electron microscopy image of monolayer $\mathrm{MoS}_{2}$ at an accelerating voltage of $80 \mathrm{kV}$ and with monochromation of the electron source. Vacancy defects are detected. 